\title{
Melissa Larner, James Peto, Nadine Monem éd., War and medecine [Guerre et médecine]
}

Londres, Black dog publishing, 2008, 256 pages.

\section{Michel Letté}

\section{OpenEdition}

Journals

Édition électronique

URL : http://journals.openedition.org/dht/617

DOI : $10.4000 /$ dht. 617

ISSN : $1775-4194$

Éditeur :

Centre d'histoire des techniques et de l'environnement du Cnam (CDHTE-Cnam), Société des élèves du CDHTE-Cnam

\section{Édition imprimée}

Date de publication : 31 mars 2009

Pagination : 222-225

ISBN : 978-2-95-30779-3-3

ISSN : 0417-8726

Référence électronique

Michel Letté, « Melissa Larner, James Peto, Nadine Monem éd., War and medecine [Guerre et médecine] ", Documents pour I'histoire des techniques [En ligne], 17/ $7^{\text {er }}$ semestre 2009, mis en ligne le 27 septembre 2010, consulté le 21 septembre 2020. URL : http://journals.openedition.org/dht/617 ; DOI : https://doi.org/10.4000/dht.617

Ce document a été généré automatiquement le 21 septembre 2020.

(C) Tous droits réservés 


\section{Melissa Larner, James Peto, Nadine Monem éd., War and medecine [Guerre et médecine]}

Londres, Black dog publishing, 2008, 256 pages.

Michel Letté

\section{RÉFÉRENCE}

Melissa Larner, James Peto, Nadine Monem éd., War and medecine [Guerre et médecine], Londres, Black dog publishing, 2008, 256 pages. 
1 La tenue à Londres du 22 novembre 2008 au 15 février 2009 de cette exposition, conjointement organisée par le Wellcome Collection et le Deutches Hygiene-Museum de Dresdes, mettait en scène le résultat d'une réflexion collective sur les techniques médicales par et pour les besoins de la guerre dite « moderne ». Elle portait plus exactement sur l'évolution de pratiques qui tentent en permanence de combler le fossé entre les capacités d'intervention et la toujours plus importante sophistication des armes permettant de mutiler toujours plus efficacement depuis une distance toujours plus grande toujours plus de masse corporelle et d'esprits. Elle proposait enfin de rendre compte des interactions qui de fait s'opèrent entre médecine militaire et civile, avec cette

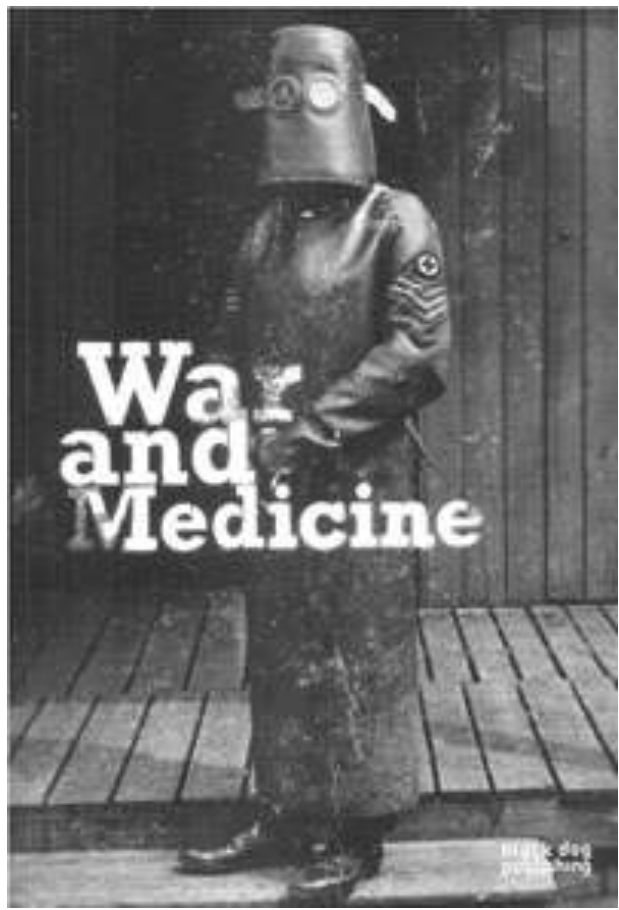
conclusion, qui se voulait sans doute optimiste, suggérant que le potentiel extraordinaire des armées pourrait bien à l'avenir servir à couvrir de par le monde les besoins civils.

2 Avec une thématique telle que celle de la réparation des corps et des psychismes dévastés par la débauche des pires techniques de violence industrielle que l'être humain est capable de s'infliger, on pouvait s'attendre à un étalage de chairs déchirées et de témoignages éprouvants de la souffrance psychologique. Il y en a en effet à profusion, mais le choc n'est pas seulement là où on l'attendait. D'abord il y a ce contraste saisissant entre l'imaginaire que suppose la pratique médicale dans un contexte de guerre (flots de sang, explosions, hurlements, chairs brûlées, gestes d'urgence et d'agitation) et le calme apparent de l'exposition, l'immobilité des objets, la lenteur des mouvements, le silence des souffrances, les gestes presque mécaniques, le rythme posé du défilement des événements et de leurs traductions techniques désincarnées. Le premier contact avec l'exposition est cependant bien physique, il bouscule les sens. C'est celui qu'impose le vrombissement épais, sourd et permanent, perçu dès le seuil de la salle, mais pleinement ressenti à l'intérieur. Ce son à l'évidence d'origine mécanique est étouffé mais intense. On imagine bien qu'il provient du moteur d'un engin de guerre énorme (un avion bombardier par exemple) juste stationné derrière la paroi de l'exposition. Il vous traverse le corps, ferait vibrer les carreaux des fenêtres de cette exposition si elle en était dotée. Surtout l'épaisseur de ces ondes sonores s'impose jusque dans les tréfonds de vos organes. Elle vous rappelle l'extrême fragilité de votre frêle carcasse, la vulnérabilité de votre corps face à des machines déshumanisées de destruction d'une incroyable efficacité. L'atmosphère invite ainsi d'emblée à cette curieuse sensation d'angoisse feutrée pour vous maintenir dans un état d'anxiété qui ne vous lâche pas tout à fait après la sortie.

3 Cette vibration qui baigne l'ensemble de l'exposition provient d'une installation proposée par un artiste contemporain, David Cotterrell, qui a projeté sur un panoramique de quatre écrans géants la scène d'une prise en charge d'un blessé. 
Immobile, relié à ses fils et tuyaux en tous genres, mais bien casé dans son carcan de matériel sophistiqué. On imagine assez bien que ce blessé est celui d'un théâtre d'opération récent, de type irakien ou afghan : tous les dispositifs déployés sont ultra modernes, les techniciens qui s'affairent sont suréquipés, les militaires bardés d'électronique, et l'un d'eux, assis à côté du blessé embarqué, manipule sur ses genoux un ordinateur portable... une vision bien éloignée des attendus de cette exposition dont le titre et l'affiche avaient plutôt suscité des images spontanées de brancards trainés dans les tranchées de la Première Guerre mondiale. L'atmosphère est assurément autre. Placé dans le noir au centre du dispositif, le visiteur fait partie d'un décor de ce qui ressemble plus au garage à peine rangé d'un bricoleur que d'une salle d'intervention chirurgicale. Les acteurs ne vous prêtent aucune attention, ne se parlent pas dans ce bruit assourdissant, mais communiquent pourtant. On trouvera entre les pages 192 et 221 du catalogue, largement illustrées, quelques explications sur la façon dont l'artiste a travaillé avec les équipes médicales sur place pour arriver à présenter ce résultat : Artists' Diary - J4MED, Op Herrick 7, 03.11.07-26.11.07. La scène est déclinée. L'artiste propose par cette reconstitution d'observer depuis d'autres registres cette même équipe médicale en action. Un simple dispositif de trois écrans situé à l'extérieur permet de montrer simultanément plusieurs traductions techniques et différentes formes de restitution d'une même réalité : l'inscription en langage informatique des quantités et de la nature des substances chimiques injectées dans ce corps inanimé défile sur un écran en couleur électronique; les images d'un superviseur distant observant silencieusement plusieurs autres écrans dans une salle où le calme est tout juste rompu par le ronronnement de la ventilation des machines informatiques; enfin, une reprise sous un autre angle de la scène première vidée de ses acteurs évoque quelque chose comme le dispositif désincarné.

4 La performance de l'artiste est le seul «écart " muséographique. Le reste demeure classique et raisonnable. L'essentiel est résumé en un agencement de dispositifs familiers : affiches, panneaux, photographies, livres et objets flanqués de leurs cartels, des écrans sur lesquels défilent des images et des témoignages. Quelques vitrines seulement. Pas d'ostentation sonore ni habillage particulier de lumière. Bref, l'ensemble est d'une grande sobriété. Couleurs et lumières sont à dominante «blanc médical », et seuls quelques blocs au fond de la salle disposés de sorte qu'ils figurent un vague effondrement viennent évoquer de façon un peu pathétique la destruction.

5 Venons-en au catalogue dont le propos pourrait sembler dispersé comme peut l'être le corps d'un soldat sur un champ de bataille. Mais il ne devait pas être si facile de rendre compte de la multitude des interrogations auxquelles le thème invite. Parmi eux, la lancinante question des progrès techniques que les conflits permettent de réaliser; la technique médicale comme pratique de guerre; les expérimentations qu'une médecine peu scrupuleuse peut avoir entreprises dans ces circonstances; l'ambiguïté de la fascination ressentie face aux réalisations dans le domaine de la réparation des corps, moins dans le domaine de la santé mentale. Une place non négligeable est cependant faite aux Post traumatic stress disorders dont on ne comprend toujours que bien peu choses à ce jour. Ce livre témoigne enfin de la difficulté que les médecins ont eue et ont aujourd'hui encore à s'imposer auprès des militaires. En dépit du rappel de cette évidence que quels que soient ses progrès la médecine militaire ne changera pas la nature des conflits, l'équivoque de ses fonctions persiste à la lecture des textes. La pratique médicale est présentée à la fois comme un instrument de guerre, mais aussi comme la promesse de son effacement. Difficile d'oublier le premier terme. L'essentiel 
de l'attention du lecteur est en fait vite attiré vers les abîmes de ce cercle infernal des ambiguïtés dans lequel sont mises les techniques dédiées à l'efficace de la destruction. En temps de guerre, la médecine a non seulement pour obligation de devoir négocier avec ce que l'être humain peut infliger de pire à ceux de son espèce, mais encore de participer à la rationalité technique par rapport à une fin qui elle n'est point équivoque : celle de vaincre l'ennemi, et de remettre pour cela à disposition de la perpétuation du massacre les blessés les moins atteints, c'est-à-dire les plus aptes à surmonter leurs blessures pour reprendre au plus vite le combat. Cette rationalité là de la guerre totale est assurément contre-intuitive.

Dix-huit auteurs - chercheurs des sciences humaines, mais aussi professionnels des musées, témoins et artiste - ont contribué à la réalisation de ce recueil original de textes, de documents, d'archives et d'images qui couvrent la période allant de la guerre de Crimée aux guerres actuelles d'Afghanistan et d'Irak.

7 La première contribution «War and medecine in the moderne era » est consacrée à la naissance de la médecine militaire. D'abord préventive, elle se concrétise par la proportion de soldats décédés du fait de la maladie désormais inférieure à celle des morts dus aux blessures. Mark Harrison, professeur d'histoire de la médecine à l'Université d'Oxford et directeur de l'unité oxonienne du Wellcome Trust for the History of Medecine explicite ainsi comment, aux termes d'une compétition technique, la médecine devient elle-même un champ de bataille sur lequel les belligérants s'affrontent.

8 Hugh Small dans "The impact of the Crimean war on the public health " prolonge le propos en s'arrêtant sur le rôle de Florence Nightingale durant cette guerre, figure à laquelle il a consacré déjà plusieurs études. Autre femme du cœur, Susane Hahn, médecin, professionnelle des musées et conférencière, s'intéresse à ses différentes blessures dans « How varied the image of heart trauma has become. The development of cardiovascular surgery during the first world war ».

"Life without arms. Carl Hermann Unthan and his motivational work with disabled veterans in Germany" de Colleen Schmitz, autre conservateur au Deutshes hygienemuseum de Dresde, retrace la mise en propagande durant plus de dix ans de la figure emblématique du vétéran surmontant la perte de ses deux bras par l'usage de ses pieds pour toutes les petites choses du quotidien. On retrouvera dans ce recueil nombre des clichés les plus frappants qui ont été utilisés dans l'exposition.

10 Ana Carden-Coyne, spécialiste des études sur la mémoire et la commémoration, est l'auteure de «Soldier's bodies in the war machine. Triage, propaganda and military medical bureaucracy, 1914-1918» qui décortique les logiques de rationalisation de la gestion des prises en charge du blessé par l'ensemble des services médicaux, depuis son chargement sur la civière jusqu'à la livraison d'une image de propagande à destination des populations civiles.

11 L'histoire des soldats indiens blessés sur le front Est et recueillis dans le pavillon de Brighton est racontée dans « Treated like flowers. The India army at the Royal pavillon hospital, Brighton, 1914-1916» par Kate Forde et Lucy Shanahan, toutes deux conservatrices de collections. Elles y décrivent la vie quotidienne et son organisation, mais rappellent aussi l'usage qui a été fait à des fins de propagande de cette mise à disposition par le Roi George V de Grande-Bretagne et d'Irlande et Empereur des Indes de son magnifique palais. 
12 La galerie de portraits en couleur de huit " gueules cassées » et " réparées » entre 1939 et 1945, proposée par Percy Hennell, n'est accompagnée que de modestes commentaires descriptifs. L'indicible s'exprime pleinement ici par l'image. Présents à l'esprit dès l'entrée dans le catalogue, on pensera plus encore à ce moment aux travaux de Sophie Delaporte'.

Dans « Suffering and the healing profession. The experience of military medicine in the first and second world wars ", Joanna Bourke, professeure d'histoire à l'Université de Londres, rappelle que la médecine militaire était plus «militaire » que "médecine». C'est aussi dans cette contribution que le lecteur verra abordée la question de l'expérimentation sur ces corps disponibles à peu de frais.

Wolfgang U. Eckart écrit sur un aspect de la seconde guerre mondiale pratiquement inexistant parmi la multitude des travaux produits sur la bataille de Stalingrad: la situation sanitaire des troupes allemandes et la gestion des services médicaux lors du siège de la ville entre le 22 novembre 1942 et le 4 février 1943. Dans "Stalingrad. Wounded bodies and souls", ce chercheur en médecine, historien et philosophe à l'Université de Munster, recompose la série des drames de cette effroyable opération militaire, des souffrances endurées par les troupes que l'impréparation et la faillite des unités sanitaires ont largement contribué à accentuer.

15 Avec l'historien britannique Ben Shephard, chercheur participant au programme Changing Character of War à Oxford, est abordée la question cruciale longtemps occultée ou négligée de la santé mentale des combattants et de tous ceux touchés d'une façon ou d'une autre par les conflits. "Why the psychiatry of war is too important to be left to psychiatrists " retrace la lente reconnaissance et la prise en considération encore difficile aujourd'hui par les autorités militaire comme civiles des souffrances et blessures affligées au psychisme².

16 William Wiesmann, Nicole Draghic et John A Parrish sont les auteurs du seul article explicitement dédié aux innovations dans le domaine de l'intervention médicale sur le champ de bataille et de la réparation des corps. Dans un panorama intitulé « Advances in modern combat causalty care with a vision to the future » et après un bref rappel des techniques passées, ils présentent, tels des technico-commerciaux fiers de leurs produits, le catalogue des quelques innovations présentes et à venir, leurs prototypes.

17 Aux cotés de tous ces articles, on trouvera quelques documents et archives présentés lors de l'exposition. Huit reproductions d'affiches conçues par Abram Games pour des campagnes de sensibilisation - dont trois de la série "You owe it to yourself, your comrades, your efficiency »- sont ainsi données à voir sans plus de commentaires. Cet artiste graphiste avait été recruté par l'armée américaine durant la Seconde Guerre mondiale pour la conception d'affiches de propagande et le recrutement, mais avait aussi produit quelques exemplaires pour le service sanitaire entre 1941 et 1946.

Suivent des retranscriptions de témoignages d'expériences vécues désormais couchées sur le papier. «Last night I dreamed of peace », est une partie des mémoires de Dang Thuy Tram, 24 ans et volontaire dans un hôpital militaire de l'armée du Viet Cong entre 1968 et 1970 . Sous le titre «Living with personal dragons in your daily life », on lira des extraits d'un autre journal dont l'auteur, aussi vétéran du Vietnam mais américain qui a souhaité conserver l'anonymat, tente d'exprimer par ces quelques mots un retour à la vie émotionnelle qu'il juge désormais impossible. Le témoignage plus récent encore de Cheryl Lynn Ruff (2005), "Ruff's war: Navy nurse on the frontline in Iraq", seule 
infirmière anesthésiste de sa compagnie, rend compte de ses expériences, non seulement de la confrontation avec les blessures endurées par ses compatriotes, mais aussi avec celles de civils iraquiens de tous les âges. Robert Acosta a seulement vingt ans au moment de son implication dans le conflit iraquien en 2003. Il fait part dans «Army specialist : Iraq war service » de son expérience de victime de l'explosion d'une grenade qu'il tenait dans sa main et de son difficile retour de héros à la vie civile. Le dernier témoignage "Open the hospital doors! » est celui d'un médecin iraquien, le docteur Mantador Taher qui exerçait dans un hôpital de Bagdad après le déclenchement de l'offensive en avril 2003 par la force multinationale. Il écrit sur le chaos, les conditions indescriptibles dans lesquelles il a dû, prisonnier d'un hôpital dévasté, continuer à néanmoins faire ce qu'il pouvait encore sans électricité ni quelconque fluide, et finalement poursuivre son activité durant plusieurs années sous la menace permanente de la mort et au milieu d'une violence qui en 2008 semblait à peine diminuer.

Comment conclure la visite de "War and Medecine» prolongée par la lecture du catalogue de l'exposition? L'expérience à laquelle elle invite devrait peut-être contribuer à favoriser la curiosité, la rencontre entre praticiens et chercheurs, les regards interdisciplinaires? Un dialogue est à l'évidence possible, en tout cas souhaitable entre médecins et spécialistes de sciences humaines et sociales, et l'expérience ici devrait tout autant inciter chercheurs en histoire des techniques et artistes, designers ou plasticiens à se parler et travailler un peu plus ensemble.

\section{NOTES}

1. Sophie Delaporte, Les Gueules cassées. Les blessés de la face de la Grande Guerre, Paris, Noêsis, 1996, 230 p. et Les médecins dans la Grande Guerre 1914-1918, Paris, Bayard, 2003, 224 p.

2. Ben Shephard, A war of nerves: Soldiers and psychiatrists in the twentieth century, Cambridge, Massachusetts, Harvard University Press, 2001, 487 p.

\section{AUTEURS}

\section{MICHEL LETTÉ}

CDHTE-Cnam 